\title{
O uso de lubrificante na citologia cervico-vaginal - Revisão baseada na evidência
}

Clara Pinto Ferreira, ${ }^{1}$ Alexandra Machado, ${ }^{2}$ Alexandra Pina, ${ }^{1}$ Ana Margarida Cruz $^{3}$

\section{RESUMO}

Objectivos: Avaliar se a utilização de lubrificante interfere no resultado da citologia cervico-vaginal. Fonte de dados: Sítios de medicina baseada na evidência e Índex de Revistas Médicas Portuguesas.

Métodos de revisão: Foi realizada uma pesquisa de artigos usando os termos MeSH vaginal smears e lubricants, publicados entre Janeiro de 2000 e Janeiro 2011; foram usadas também algumas referências cruzadas. Foi utilizada a Oxford Centre for Evidence-based Medicine - Levels of Evidence para avaliação da qualidade dos estudos e posterior atribuição de níveis de evidência e forças de recomendação.

Resultados: Dos 41 artigos identificados, foram seleccionados oito: sete ensaios clínicos aleatorizados e controlados (ECAC) e um estudo retrospectivo. Seis dos estudos (três de nível de evidência 1 b e três de nível 2 b) concluem que o uso de lubrificante na citologia cervico-vaginal não altera a qualidade do resultado das amostras citológicas obtidas. Os restantes dois artigos (nível 2 b) apontam para uma interferência do lubrificante no resultado citológico.

Conclusões: Os conhecimentos actuais relativos a este tema são escassos e os estudos apresentam protocolos heterogéneos quanto ao desenho e técnicas usadas. No entanto, os estudos cujas metodologias mais se aproximam da prática clínica apontam para que o uso de uma pequena quantidade de lubrificante aquoso não interfira com o resultado da citologia cervico-vaginal (Força de Recomendação A), pelo que poderá ser um procedimento a ter em conta nas situações avaliadas pelo clínico como sendo de interesse para a mulher.

Palavras-chave: Lubrificantes; Esfregaço Vaginal.

\section{INTRODUÇÃO}

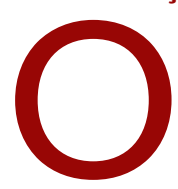

cancro do colo do útero é o sétimo cancro mais prevalente a nível mundial, sendo o segundo mais frequente no sexo feminino; de acordo com dados de 2002, causou a morte a cerca de 275 mil mulheres. ${ }^{1}$ Dados portugueses de 2004 apontam para o aparecimento de cerca de mil novos casos por ano e indicam que esta doença é responsável pela morte de, em média, uma mulher por dia. ${ }^{2}$ A implementação de sistemas de rastreio organi-

\footnotetext{
${ }^{1}$ Internas de Medicina Geral e Familiar na Unidade de Saúde Familiar Horizonte, ACES de Matosinhos

${ }^{2}$ Interna de Medicina Geral e Familiar na Unidade de Saúde Familiar Lagoa,ACES de Matosinhos

${ }^{3}$ Médica de Família, Unidade de Cuidados de Saúde Personalizados de Grijó,ACES de Espinho/Gaia
}

zado e de base populacional em alguns países como Canadá e países do Norte da Europa tem-se reflectido numa diminuição da incidência e mortalidade desta patologia. $^{3}$

A técnica de citologia cervico-vaginal é o método de base para o rastreio do cancro do colo do útero. É um procedimento sentido como desconfortável por muitas mulheres, o que poderá determinar o seu não comparecimento às consultas de rastreio. ${ }^{4-5} \mathrm{~A}$ utilização de lubrificante para facilitar a introdução do espéculo é tida empiricamente como uma forma de minimizar o desconforto sentido pela mulher durante o exame. Em última instância, esta facilitação pode contribuir para uma maior adesão ao rastreio, levando a uma diminuição da incidência da doença. Por outro lado, o uso de lubrificante é controverso e desencorajado por vários autores,,$^{5-6} \mathrm{com}$ base no pressuposto de que poderá al- 
terar a qualidade das citologias, mas sem um fundamento científico concreto.

$\mathrm{O}$ rastreio do cancro do colo do útero assume-se como uma das actividades preventivas principais em Medicina Geral e Familiar (MGF), com resultados comprovados de ganhos em saúde para a população feminina. A melhoria da qualidade da técnica de rastreio em termos de conforto poderá ser encarada como uma mais-valia na adesão das mulheres a este procedimento. O objectivo deste estudo foi rever a evidência disponível sobre a interferência do uso de lubrificante no resultado das citologias do colo do útero.

\section{MÉTODOS}

Foi realizada uma pesquisa sistemática nas bases de dados UpToDate, BMJ Clinical Evidence, EBM Journal, TRIP Database, Cochrane Library, DARE, Bandolier, National Guideline Clearinghouse, NeLH Guidelines Finder, Medline e Índex de Revistas Médicas Portuguesas, de normas de orientação clínica, revisões sistemáticas, meta-análises e estudos originais, publicados entre Janeiro de 2000 e Janeiro de 2011, em inglês, francês, espanhol e português, utilizando os termos MeSH vaginal smears e lubricants.

Os critérios definidos para inclusão de artigos nesta revisão foram: a) População: mulheres que realizaram rastreio do cancro do colo do útero por técnica convencional ou em meio líquido; b) Intervenção: utilização de lubrificante aquando da colheita da amostra para citologia; c) Comparação: utilização de água ou ausência de qualquer tipo de lubrificante aquando da colheita da amostra para citologia; d) Resultado: alteração da qualidade das amostras com comprometimento do resultado citológico. A existência prévia de patologia cervical e a presença de infecção vaginal activa foram critérios de exclusão.

Para avaliar a qualidade dos estudos e posterior atribuição do nível de evidência (NE) e forças de recomendação (FR) foi utilizada a escala Oxford Centre for Evidence-based Medicine - Levels of Evidence. ${ }^{7}$ Esta taxonomia subdivide a qualidade do estudo em cinco Níveis de Evidência (Quadro I) e a Força de Recomendação em quatro graus, como descrito no Quadro II.

\section{RESULTADOS}

Da pesquisa inicial obtiveram-se 41 artigos. Destes

\begin{tabular}{|c|c|}
\hline \multicolumn{2}{|r|}{$\begin{array}{l}\text { QUADRO I. Níveis de Evidência (Oxford Centre for } \\
\text { Evidence-based Medicine). }\end{array}$} \\
\hline NE & Significado \\
\hline $1 \mathrm{a}$ & $\begin{array}{l}\text { Revisões sistemáticas (com homogeneidade) de } \\
\text { ensaios clínicos aleatorizados (ECA) }\end{array}$ \\
\hline $1 \mathrm{~b}$ & ECA individuais (com intervalo de confiança estreito) \\
\hline 1c & «Tudo ou nada» \\
\hline $2 a$ & $\begin{array}{l}\text { Revisões sistemáticas (com homogeneidade) de } \\
\text { estudos cohort }\end{array}$ \\
\hline $2 b$ & $\begin{array}{l}\text { Estudos cohort individuais (incluindo ECA fraca } \\
\text { qualidade) }\end{array}$ \\
\hline $2 c$ & Pesquisa de «outcome»; estudos ecológicos \\
\hline 3a & $\begin{array}{l}\text { Revisões sistemáticas (com homogeneidade) de } \\
\text { estudos caso-controlo }\end{array}$ \\
\hline $3 b$ & Estudos individuais de caso-controlo \\
\hline 4 & $\begin{array}{l}\text { Série de casos (e estudos cohort e caso-controlo de } \\
\text { fraca qualidade) }\end{array}$ \\
\hline 5 & $\begin{array}{l}\text { Opinião de peritos sem comentário crítico explícito, } \\
\text { ou baseada na fisiologia ou em pesquisa de fraca } \\
\text { qualidade }\end{array}$ \\
\hline
\end{tabular}

NE: Nível de Evidência. ECA: Ensaio clínico aleatorizado.

QUADRO II. Forças de Recomendação (Oxford Centre for Evidence-based Medicine).

FR Significado

A Estudos consistentes de nível 1

B Estudos consistentes de nível 2 ou 3 ou extrapolações de estudos nível 1

C Estudos de nível 4 ou extrapolações de estudos nível 2 ou 3

D Estudos de nível 5 ou estudos inconclusivos ou inconsistentes de qualquer nível

FR: Força de Recomendação.

foram excluídos os artigos em que se verificou discordância com o objectivo da revisão, aqueles que não cumpriam os critérios de inclusão e os artigos repetidos.

Foram seleccionados oito artigos, ${ }^{8-15}$ sete dos quais representam ensaios clínicos controlados e aleatoriza- 


\begin{tabular}{|c|c|c|c|c|}
\hline Referência & $\begin{array}{l}\text { População } \\
\text { (n) }\end{array}$ & Intervenção & Conclusões & NE \\
\hline $\begin{array}{l}\text { Amies AM } \\
\text { et al. } 2002^{8}\end{array}$ & 8534 & $\begin{array}{l}\mathrm{HR}^{\circledR} \text { Lubricating } \\
\text { Jelly («dime-sized») } \\
\text { vs água } \\
\text { Citologia convencional }\end{array}$ & $\begin{array}{l}\text { Taxa de amostras insatisfatórias: com lubrificante } 1,4 \% \text { vs sem } \\
\text { lubrificante } 1,3 \% \text {, OR 1,1 (IC 95\%; 0,6-2,0) } \\
\text { Sem diferenças significativas na percentagem de citologias com } \\
\text { resultado anatomo-patológico alterado (ASCUS, LIBG) }\end{array}$ & $1 b$ \\
\hline $\begin{array}{l}\text { Griffith WF } \\
\text { et al. } 2005^{9}\end{array}$ & 3460 & $\begin{array}{l}\text { Surgilube }{ }^{\circledR} \\
\text { («dime-sized») vs água } \\
\text { Citologia convencional }\end{array}$ & $\begin{array}{l}\text { Taxa de citologias insatisfatórias semelhante: } 1,1 \% \text { com lubrificante } \\
\text { vs } 1,5 \% \text { com água, OR } 0,74 \text { (IC } 95 \% ; 0,42-1,35)\end{array}$ & $1 b$ \\
\hline $\begin{array}{l}\text { Hathaway JK } \\
\text { et al. } 2006^{10}\end{array}$ & 200 & $\begin{array}{l}\text { Controlo vs amostra } \\
\text { contaminada com } \\
0,5 \mathrm{ml} \text { de Surgilube }{ }^{\circledR} \\
\text { na mesma mulher } \\
\text { Citologia em meio } \\
\text { líquido }\end{array}$ & $\begin{array}{l}\text { Contaminação da amostra por lubrificante não afecta a interpretação } \\
\text { citológica. } \\
\text { A diferença entre os resultados de citologias com e sem lubrificante } \\
\text { é de } 7,5 \% \text { (IC } 95 \% ; 4,6-12,1 \% \text { ) }\end{array}$ & $1 b$ \\
\hline $\begin{array}{l}\text { Charoenkwan } \\
\text { K et al. } 2008^{11}\end{array}$ & 1334 & $\begin{array}{l}\text { Controlo vs colo } \\
\text { contaminado com } \\
\mathrm{K}-\mathrm{Y}^{\circledR} \text { Jelly (numa } \\
\text { extensão de } 1-1,5 \mathrm{~cm} \\
\text { de gel), na mesma } \\
\text { mulher } \\
\text { Citologia convencional }\end{array}$ & $\begin{array}{l}\text { Taxa de citologias insatisfatórias superior com aplicação de } \\
\text { lubrificante }(12,1 \% \text { vs } 1,7 \% ; p<0,01)\end{array}$ & $2 b$ \\
\hline $\begin{array}{l}\text { Harer WB } \\
\text { et al. } 2002^{12}\end{array}$ & 182 & $\begin{array}{l}2-3 \mathrm{~cm}^{3} \text { de Surgilube }{ }^{\circledR} \\
\text { vs água (aplicados no } \\
\text { intróito) } \\
\text { Citologia convencional }\end{array}$ & $\begin{array}{l}\text { Lubrificação do intróito vaginal e das superfícies externas do } \\
\text { espéculo não afectam a qualidade do esfregaço citológico (taxa de } \\
\text { insatisfatórios } 2,1 \% \text { com Surgilube }{ }^{\circledR} \text { vs } 2,2 \% \text { com controlo, } p=0,528 \text { ) }\end{array}$ & $2 b$ \\
\hline $\begin{array}{l}\text { Holton T } \\
\text { et al. } 2008^{13}\end{array}$ & 110 & $\begin{array}{l}0,1-0,5 \mathrm{~g} \text { de } \mathrm{K}-\mathrm{Y}^{\circledR} \\
\text { Jelly vs } 0,1-0,5 \mathrm{~g} \text { de } \\
\text { Aquagel}{ }^{\circledR} \text { vs sem } \\
\text { lubrificante } \\
\text { Citologia em meio } \\
\text { líquido }\end{array}$ & $\begin{array}{l}\text { A contaminação directa da amostra citológica com lubrificante pode } \\
\text { resultar numa celularidade reduzida }(p<0,001)\end{array}$ & $2 b$ \\
\hline $\begin{array}{l}\text { Gilson } M \\
\text { et al. } 2006^{14}\end{array}$ & 70 & $\begin{array}{l}\text { Sem lubrificante vs } \\
2,7 \mathrm{~g} \text { de gel aquoso } \\
\text { nas lâminas do } \\
\text { espéculo, na } 2 .^{\text {a }} \\
\text { amostra na mesma } \\
\text { mulher } \\
\text { Citologia convencional }\end{array}$ & $\begin{array}{l}\text { Pequena quantidade de gel lubrificante no espéculo não altera a } \\
\text { citologia cervico-vaginal convencional }(p=0,5)\end{array}$ & $2 b$ \\
\hline
\end{tabular}

NE: Nível de Evidência. OR: Odds ratio. ASCUS: Sigla em inglês para células escamosas atípicas de significado indeterminado. LIBG: Lesão intraepitelial de baixo grau. 


\section{QUADRO IV. Estudo retrospectivo.}

\begin{tabular}{llll} 
Referência & População $(\mathbf{n})$ & Análise & Conclusões \\
\hline $\begin{array}{l}\text { Tavernier LA } \\
\text { et al. } 2003^{15}\end{array}$ & 615 & $\begin{array}{l}\text { Questionado o uso de lubrificante } \\
\text { (sem observação directa) } \\
\text { Citologia convencional }\end{array}$ & $\begin{array}{l}\text { O uso de uma camada fina de lubrificante aquoso } \\
\text { não compromete a qualidade da citologia }\end{array}$ \\
\hline
\end{tabular}

NE: Nível de Evidência.

dos (ECAC) (Quadro III) e um estudo retrospectivo (Quadro IV).

Foi atribuído nível de evidência $1 b$ aos estudos de Amies et al,$^{8}$ Griffith WF et al ${ }^{9}$ e Hathaway et al,$^{10}$ todos eles ECAC individuais com intervalo de confiança estreito, população de dimensão significativa e com metodologia e resultados bem descritos e compreensíveis. Nos primeiros dois estudos foi aplicada uma pequena quantidade («dime-sized $»^{1}$ ) de gel lubrificante aquoso na superfície externa do espéculo quando da realização da citologia cervico-vaginal em lâmina (convencional), com utilização de água como lubrificante no grupo controlo. Relativamente ao estudo de Griffith WF, e atendendo à metodologia, as colheitas foram realizadas por diferentes profissionais de saúde, com experiências também díspares, pelo que esta variabilidade de profissionais pode causar um viés e ser uma limitação do estudo. No terceiro ensaio, duplamente cego e com recurso a citologia em meio líquido, foram colhidas duas amostras citológicas para cada mulher, aplicando-se posteriormente e de forma aleatória uma pequena quantidade de lubrificante directamente na espátula $\mathrm{e}$ escova, colocando-as de seguida no meio líquido destinado a análise, usando-se como controlo amostras não «contaminadas». O procedimento deste estudo em nada espelha a prática clínica real, o que dificulta a transposição das suas conclusões para a prática médica. Estes três estudos concluem que a aplicação de uma pequena quantidade de lubrificante aquoso não interfere na interpretação nem altera os resultados da citologia cervico-vaginal.

\footnotetext{
1. A quantidade de gel aplicada pelos investigadores foi descrita como "dime-sized", sendo o termo comparativo a moeda de dez cêntimos americana, que tem cerca de $17 \mathrm{~mm}$ de diâmetro e 2,268 g de peso.
}

Aos restantes cinco estudos foi atribuído um nível de evidência $2 b$. No estudo duplamente cego de Charoenkwan $\mathrm{K}$ et $a l^{11}$ concluiu-se que a aplicação de lubrificante no colo uterino pode comprometer a avaliação da citologia cervico-vaginal convencional; neste ensaio, houve uma primeira colheita de células cervicais, seguindo-se a aplicação directa de lubrificante no colo uterino, procedimento esse que não espelha a prática clínica real e que poderá ter determinado, por efeito directo de barreira física, uma colheita celular deficitária. Assim, embora se trate de um ensaio com uma amostra considerável, apresenta uma metodologia de fraca qualidade.

Harer WB e colaboradores ${ }^{12}$ afirmam no seu estudo que a lubrificação do intróito vaginal e das superfícies externas do espéculo com lubrificante facilitam o exame e não afectam a qualidade do esfregaço citológico, efectuado pelo método convencional com recurso a espátula e escova. A amostra utilizada foi, no entanto, demasiado reduzida para poder obter uma significância estatística para esta conclusão.

No ensaio de Holton T et al, ${ }^{13}$ os investigadores procederam à aplicação directa de quantidades crescentes de dois tipos de lubrificante no meio líquido da amostra citológica, tendo como controlo amostras não «contaminadas», e concluíram que esta acção pode resultar numa celularidade reduzida. Neste estudo, verificou-se que, com o lubrificante $\mathrm{K}-\mathrm{Y}^{\circledast}$ Jelly, a contaminação apenas se deu com as quantidades mais altas de lubrificante $(0,4$ e $0,5 \mathrm{~g})$, enquanto para o lubrificante Aquagel $^{\circledR}$ a contaminação aconteceu com qualquer quantidade de lubrificante. É ainda importante referir que o efeito provocado pelo lubrificante é aquando da preparação da amostra no laboratório, preparação que não acontece de igual forma na lâmina convencional. A reduzida dimensão da amostra de lâminas foi, no entan- 
to, uma grande limitação neste estudo, uma vez que, para cada tipo e quantidade de lubrificante, apenas foram analisadas dez amostras, que foram comparadas com amostras de controlo que não pertenciam à mesma mulher. Para além deste facto, a forma como foram obtidos e apresentados os resultados é pouco clara.

Gilson $\mathrm{M}$ et $a l^{14}$ realizaram um estudo com uma amostra de pequena dimensão, no qual foram efectuadas para cada mulher duas citologias convencionais consecutivas, com espátula e escova: a primeira sem lubrificante; a segunda com lubrificante aquoso para os casos ou novamente sem lubrificante para os controlos. Concluíram que uma pequena quantidade de gel lubrificante no espéculo não altera a citologia cervicovaginal. Apesar de ser um estudo bem desenhado, o seu nível de evidência é limitado pela dimensão da amostra utilizada.

O estudo conduzido por Tavernier LA et $a l^{15}$ é de carácter retrospectivo, com base em registos médicos que incluíam uma análise anatomopatológica relatando a adequação da amostra citológica, e na informação dada pelo médico em resposta a um questionário sobre a sua prática de utilização ou não utilização de lubrificante. $\mathrm{Na}$ análise concluíram que o uso de uma camada fina de lubrificante aquoso no espéculo não compromete a adequação ou a interpretação da citologia convencional, por não terem encontrado diferenças estatisticamente significativas entre o uso de gel lubrificante, o uso de água para lubrificar e a não lubrificação. Este tipo de desenho é limitado pelo possível viés de memória dos clínicos e pelo facto de não se tratar de um ensaio clínico aleatorizado e com ocultação.

\section{CONCLUSÕES}

O reduzido número de estudos encontrados apresenta elevada heterogeneidade, sobretudo em termos metodológicos, relativamente ao desenho e técnicas utilizadas, como por exemplo a quantidade de lubrificante usado e a técnica de colheita da citologia. Todos eles apresentam resultados relativos ao uso de lubrificantes do tipo aquoso. Os estudos cujas metodologias mais se aproximam da prática clínica real apontam para que não haja interferência do lubrificante no resultado das citologias do colo do útero. No entanto, é relevante ter em consideração que alguns autores consideram ainda a possibilidade do lubrificante diminuir a acção dos agentes fixadores após a colheita por citologia convencional.

Embora não fosse um dos objectivos deste trabalho, é importante realçar que o único estudo incluído nesta revisão que avaliou o grau de desconforto da mulher não comprovou uma melhoria com o uso de lubrificante, contrariando a noção empírica de que poderá diminuir o desconforto provocado na mulher pelo procedimento de colheita da citologia cervico-vaginal.

Em conclusão, e de acordo com os dados obtidos, pode afirmar-se que o uso de uma pequena quantidade de lubrificante aquoso não interfere com o resultado da citologia cervico-vaginal (Força de Recomendação A), pelo que poderá ser um procedimento a ter em conta nas situações avaliadas pelo clínico como sendo de interesse para a mulher. Esta recomendação é suportada pelos três estudos com nível de evidência elevado (1b), elaborados com rigor científico e metodologias semelhantes, onde participou um elevado número de mulheres, sendo que dois deles reflectem a prática clínica na técnica de colheita do material para análise.

Apesar da questão clínica inicial desta revisão poder ser vista como tendo um resultado orientado para a doença e não para a doente, a sua implicação prática poderá ser útil por trazer uma opção viável para a realização de citologias de rastreio do cancro do colo do útero em mulheres que de outra forma poderiam não permitir esse procedimento. A decisão pela sua utilização, que a ter lugar dever-se-á referir a um lubrificante do tipo aquoso, deve ser efectuada com base no critério clínico do médico, orientado para cada mulher.

No futuro, serão necessários mais estudos cientificamente rigorosos, com metodologia mais homogénea e maior poder estatístico.

Serão ainda pertinentes estudos que avaliem se a quantidade de gel interfere com a qualidade da amostra, bem como a eventual redução do desconforto das mulheres pela utilização de lubrificante na realização da citologia cervico-vaginal.

\section{REFERÊNCIAS BIBLIOGRÁFICAS}

1. Programa de Rastreio do Cancro do Colo do Útero na Região Norte. Porto: ARS Norte; 2009.

2. Consenso Nacional do Cancro Ginecológico. Lisboa: Sociedade Portuguesa de Ginecologia; 2007.

3. Manual Executivo do Programa de Rastreio do Cancro do Colo do Útero 
na Região Norte. Porto: ARS Norte; 2009.

4. Byrd TL, Peterson SK, Chavez R, Heckert A. Cervical cancer screening beliefs among young Hispanic women. Prev Med 2004 Feb; 38 (2): 192 7.

5. Harmanli $O$, Jones KA. Using lubricant for speculum insertion. Obstet Gynecol 2010 Aug; 116 (2 Pt 1): 415-7.

6. European guidelines for quality assurance in cervical cancer screening. Lyon: International Agency for Research on Cancer; 2008.

7. Oxford Centre for Evidence-based Medicine - Levels of Evidence. March 2009. Disponível em: http://www.cebm.net/index.aspx?o=1025 [acedido em 18/04/2011].

8. Amies AM, Miller L, Lee SK, Koutsky L. The effect of vaginal speculum lubrication on the rate of unsatisfactory cervical cytology diagnosis. Obstet Gynecol 2002 Nov; 100 (5 Pt 1): 889-92.

9. Griffith WF, Stuart GS, Gluck KL, Heartwell SF. Vaginal speculum lubrication and its effects on cervical cytology and microbiology. Contraception 2005 Jul; 72 (1): 60-4.

10. Hathaway JK, Pathak PK, Maney R. Is liquid-based pap testing affected by water-based lubricant? Obstet Gynecol 2006 Jan; 107 (1): 66-70.

11. Charoenkwan K, Ninunanahaeminda K, Khunamornpong S, Srisomboon J, Thorner PS. Effects of gel lubricant on cervical cytology. Acta Cytol 2008 Nov-Dec; 52 (6): 654-8.

12. Harer WB, Valenzuela G Jr, Lebo D. Lubrication of the vaginal introitus and speculum does not affect Papanicolaou smears. Obstet Gynecol 2002 Nov; 100 (5 Pt 1): 887-8.

13. Holton T, Smith D, Terry M, Madgwick A, Levine T. The effect of lubricant contamination on ThinPrep ${ }^{\circledR}$ (Cytyc) cervical cytology liquid-based preparations. Cytopathology 2008 Aug; 19 (4): 236-43.

14. Gilson M, Desai A, Cardoza-Favarato G, Vroman P, Thornton JA. Does gel affect cytology or comfort in the screening papanicolaou smear? J Am Board Fam Med 2006 Jul-Aug; 19 (4): 340-4.

15. Tavernier LA, Connor PD, Gates D. Water versus gel lubricant for cervical cytology specimens. J Fam Pract 2003 Sep; 52 (9): 701-4.

\section{CONFLITOS DE INTERESSE}

As autoras declaram ausência de conflitos de interesses e financiamento do estudo.

\author{
ENDEREÇO PARA CORRESPONDÊNCIA \\ Clara Pinto Ferreira \\ R. Alfredo Cunha 365 \\ 4450-021 Matosinhos \\ E-mail: clarapf.mgf@gmail.com
}

Recebido em 18/04/2011

Aceite para publicação em 27/11/2011

\section{ABSTRACT}

\section{CERVICAL CYTOLOGY AND THE USE OF LUBRICANTS: AN EVIDENCE BASED REVIEW}

Objectives: To review recent evidence on the effect of lubricant on the cytological result obtained from cervical smears.

Data sources: Evidence-based medicine websites and Índex de Revistas Médicas Portuguesas.

Methods: A search for articles using the MeSH terms vaginal smears and lubricants published between January 2000 and January 2011 was conducted. Citations were also searched. The Oxford Centre for Evidence-based Medicine - Levels of Evidence was used to assess the quality of studies, the levels of evidence and the strength of recommendations.

Results: Of the 41 articles identified, eight were selected for this review. These included seven randomized controlled trials (RCT) and one retrospective study. Six of them (three with evidence level $1 \mathrm{~b}$ and three with level $2 \mathrm{~b}$ ) conclude that lubricant does not affect the quality of the cytological samples obtained. The remaining two articles (level $2 \mathrm{~b}$ ) found interference with the cytological result by lubricant.

Discussion: The use of lubricant in obtaining cervical smears is controversial and is usually not advised. Current knowledge in this area is limited. Clinical trials are heterogeneous with regard to design and techniques used. Trials with protocols that most resemble current clinical practice demonstrate no interference by lubricant with the quality of cytological samples. There is evidence that a small amount of water-based lubricant does not compromise the quality of cervical cytology results (Strength of recommendation A).

Keywords: Lubricants; Vaginal Smears. 